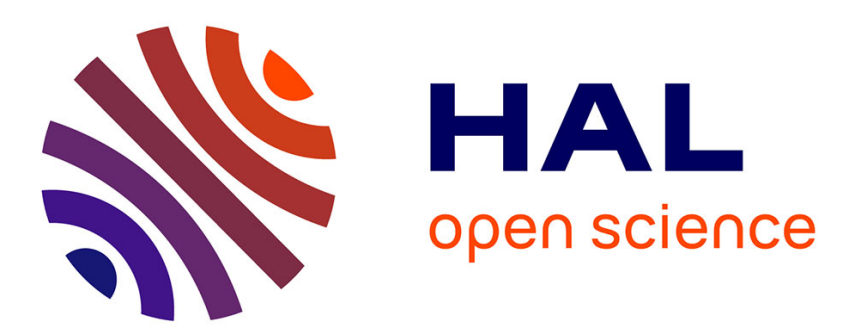

\title{
Effect of Strain Dependent Cohesive Zone Model on Predictions of Interface Crack Growth
}

\author{
V. Tvergaard, J. Hutchinson
}

\section{To cite this version:}

V. Tvergaard, J. Hutchinson. Effect of Strain Dependent Cohesive Zone Model on Predictions of Interface Crack Growth. Journal de Physique IV Proceedings, 1996, 06 (C6), pp.C6-165-C6-172. 10.1051/jp4:1996616 . jpa-00254444

HAL Id: jpa-00254444

https://hal.science/jpa-00254444

Submitted on 1 Jan 1996

HAL is a multi-disciplinary open access archive for the deposit and dissemination of scientific research documents, whether they are published or not. The documents may come from teaching and research institutions in France or abroad, or from public or private research centers.
L'archive ouverte pluridisciplinaire HAL, est destinée au dépôt et à la diffusion de documents scientifiques de niveau recherche, publiés ou non, émanant des établissements d'enseignement et de recherche français ou étrangers, des laboratoires publics ou privés. 


\title{
Effect of Strain Dependent Cohesive Zone Model on Predictions of Interface Crack Growth
}

\author{
V. Tvergaard and J.W. Hutchinson* \\ Department of Solid Mechanics, Technical University of Denmark, Lyngby, Denmark \\ * Division of Applied Sciences, Harvard University, Cambridge, MA, U.S.A.
}

\begin{abstract}
Crack growth along an interface joining an elastic-plastic solid to a solid that does not yield plastically is studied numerically, accounting for mixed mode loading under conditions of small scale yielding. The fracture process is represented in terms of a cohesive zone model, for which the work of separation per unit area and the peak stress required for separation are basic parameters; but where also a plastic strain effect on the fracture process is incorporated. This additional effect is included to model accelerated void nucleation and growth at the interface, resulting from intense plastic straining just in front of the crack tip.
\end{abstract}

\section{INTRODUCTION}

Dissipation in the plastic zone surrounding a crack tip gives a major contribution to the work of fracture of ductile materials. Thus, the macroscopic work of fracture is typically much larger than the work absorbed by the local fracture process required to separate the crack surfaces. This role of plastic dissipation in amplifying crack growth resistance has been studied by Tvergaard and Hutchinson [1] for mode 1 crack growth under conditions of small scale yielding, using a cohesive zone model to represent the fracture process at the crack tip. Also the effect of a T-stress on mode 1 crack growth resistance has been studied by this method [2].

The analyses. $[1,2]$ show that with a peak stress $\hat{\sigma}$ in the traction-separation law representing the fracture process, and an initial yield stress $\sigma_{Y}$, the predicted fracture toughness depends strongly on the ratio $\hat{\sigma} / \sigma_{Y}$. For a low value of the ratio $\hat{\sigma} / \sigma_{Y}$, plasticity does not amplify the crack growth resistance, whereas for a larger value the macroscopic work of fracture can be increased by a factor 10 or more. But the value of $\hat{\sigma} / \sigma_{Y}$ can be so large that the peak stress during blunting of a stationary crack does not reach the level $\tilde{\hat{\sigma}}$, so that no crack advance is predicted. However, in cases where void growth to coalescence is part of the crack growth mechanism, the intense deformations near the tip amplify the void growth and may also lead to nucleation of new voids, effectively lowering the peak separation stress. To incorporate such effects, Tvergaard and Hutchinson [3] and Tvergaard [4] have used a modified traction-separation law so that plastic straining gives a reduction of the peak stress for separation (see also Tvergaard [5,6]). This modified model allows for a better representation of very tough materials with a high value of the tearing modulus defined in [7]. Since plastic straining near the tip is sensitive to the element size, this modified model displays some mesh dependence.

For a crack growing along an interface joining an elastic-plastic solid to a solid that does not yield plastically a number of experiments have shown a strong dependence on the mode of loading, such that the nearmode 2 toughness can be as much as a factor of 10 higher than the near mode 1 toughness (Cao and Evans [8], Liechti and Chai [9], Thouless [10], O'Dowd et al. [11]). In an analysis of interface crack growth, using a cohesive zone model analogous to that in $[1,2]$ to represent the fracture process, Tvergaard and Hutchinson 
[12] have shown that the strong dependence of interface toughness on the relative proportion of mode 2 to mode 1 is directly predicted as a result of plastic yielding in the ductile solid on one side of the interface.

In the present paper, the effect of the plastic strain dependent cohesive zone model is investigated for the case of interface crack growth. The elastic substrate is approximated as rigid, and thus the elastic fields used as remote boundary conditions in the small scale yielding analysis must account for a non standard bi-material interface singularity. Mixed mode loading conditions are considered, and the computed interface toughness is related to the local mode mixity at a point near the edge of the plastic zone surrounding the crack tip. The results are related to those found in [12], but the main interest here is in considering cases where the initial peak stress $\hat{\sigma}$ in the traction-separation law is so high that subsequent reductions due to plastic straining play an important role.

\section{SMALL_SCALE YIELDING FORMULATION AND NUMERICAL METHOD}

The plane strain interface crack problem is solved for conditions of small-scale yielding with mixed mode loading, as in [12]. Thus, on an outer boundary with radius $A_{0}$, measured from the initial crack tip, displacements are specified corresponding to the elastic crack tip fields.

The elastic crack tip singularity field (Rice [13]) governing the remote field of a semi-infinite interface crack has tractions acting on the interface which are given in terms of the two stress intensity factor components, $K_{1}$ and $K_{2}$, by

$$
\sigma_{22}+i \sigma_{12}=\left(\mathrm{K}_{1}+\mathrm{iK}_{2}\right)(2 \pi \mathrm{r})^{-1 / 2} \mathrm{r}^{-\mathrm{i} \epsilon}
$$

Here, $\mathrm{r}$ is the distance from the tip, $\mathrm{i}=\sqrt{-1}, \epsilon$ is the oscillation index

$$
\epsilon=\frac{1}{2 \pi} \ln \left(\frac{1-\beta}{1+\beta}\right)
$$

and $\beta$ is the second Dundurs' parameter

$$
\beta=\frac{1}{2} \frac{\mu\left(1-2 v_{s}\right)-\mu_{s}(1-2 v)}{\mu\left(1-v_{s}\right)+\mu_{s}(1-v)}
$$

with $\mu=E /(2(1+v))$ and $\mu_{s}=E_{s} /\left(2\left(1+v_{s}\right)\right)$, where $E_{s}, v_{s}$ are the elastic constants for the elastic substrate, and $\mathrm{E}, v$ are the elastic constants for the elastic-plastic material. The relation between the energy release rate and the magnitude $|\mathrm{K}|$ of stress intensity factors is

$$
\mathrm{G}=\frac{1}{2}\left(1-\beta^{2}\right)\left[\frac{1-v^{2}}{\mathrm{E}}+\frac{1-v_{s}^{2}}{E_{\mathrm{s}}}\right]|\mathrm{K}|^{2}, \quad|\mathrm{~K}|=\sqrt{\mathrm{K}_{1}^{2}+\mathrm{K}_{2}^{2}}
$$

With a reference length $\mathrm{L}$ chosen to characterize the remote field an $\mathrm{L}-$ dependent measure of mode mixity $\psi$ is defined by

$$
\tan \psi=\frac{\operatorname{Im}\left[\left(K_{1}+i K_{2}\right) L^{i \epsilon}\right]}{\operatorname{Re}\left[\left(K_{1}+i K_{2}\right) L^{i \epsilon}\right]}
$$

which reduces to the more familiar measure, $\tan \psi=\mathrm{K}_{2} / \mathrm{K}_{1}$, when $\epsilon=0$. By using (2.1) in (2.5) is is seen that $\tan \psi=\sigma_{12} / \sigma_{22}$ at $\mathrm{r}=\mathrm{L}$ on the interface. Thus, $\psi$ measures the relative proportion of shear to normal stress on the interface a distance $L$ from the tip, as predicted by the elastic solution. The displacement components associated with the singularity field in the upper half-space $(\theta>0)$ are given by 


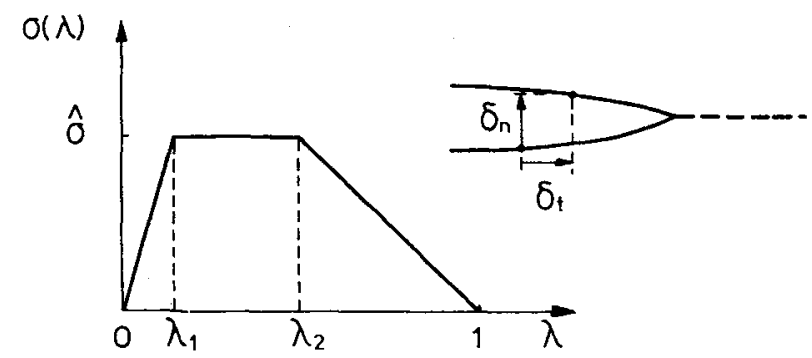

Fig. 1. Traction-generalized displacement relation used for interface separation.

$\mu\left(\mathrm{u}_{1}+\mathrm{i} \mathrm{u}_{2}\right)=\frac{|\mathrm{K}| \mathrm{r}^{1 / 2}}{2 \sqrt{2 \pi} \cosh (\pi \epsilon)}\left\{\frac{(3-4 v) \mathrm{e}^{\mathrm{i} \theta / 2+\epsilon(\theta-\pi)-\mathrm{i} \psi}}{1-2 \mathrm{i} \epsilon}-\frac{\mathrm{e}^{-\mathrm{i} \theta / 2-\epsilon(\theta-\pi)-\mathrm{i} \psi}}{1-2 \mathrm{i} \epsilon}-\mathrm{i} \sin \theta \mathrm{e}^{\mathrm{i} \theta / 2+\epsilon(\theta-\pi)+\mathrm{i} \bar{\psi}}\right\}$

where $\bar{\psi}$ depends on $r$ according to

$$
\bar{\psi}=\psi+\epsilon \ln (\mathrm{r} / \mathrm{L})
$$

The expression for the components in the lower half-space is similar in form.

In specifying the small-scale yielding problem, the displacement components $u_{1}$ and $u_{2}$ given by (2.6) are imposed remotely on a boundary at $r=A_{0}$, where $A_{0}$ is typically several times $L$. The remote loading is thus specified by $|K|, \Psi$ and $L$.

A model of Needleman [14] describing debonding under purely normal separation was extended by Tvergaard [15] to also account for tangential separation, and a version of this extended model was applied by Tvergaard and Hutchinson [12] to represent the interface fracture process. As illustrated in Fig. $1, \delta_{n}$ and $\delta_{t}$ are the normal and tangential components of the relative displacements of the crack faces across the interface. Critical values of these displacement components are denoted $\delta_{n}^{c}$ and $\delta_{t}^{c}$, and a nondimensional crack separation measure is defined as

$$
\lambda=\sqrt{\left(\delta_{n} / \delta_{n}^{c}\right)^{2}+\left(\delta_{t} / \delta_{t}^{c}\right)^{2}}
$$

such that the tractions drop to zero when $\lambda=1$. With $\sigma(\lambda)$ displayed in Fig. 1, and a traction potential defined [12] as

$$
\Phi\left(\delta_{\mathrm{n}}, \delta_{\mathrm{t}}\right)=\delta_{\mathrm{n}}^{\mathrm{c}} \int_{0}^{\lambda} \sigma\left(\lambda^{\prime}\right) \mathrm{d} \lambda^{\prime}
$$

the normal and tangential components of the traction acting on the interface in the fracture process zone can be written as

$$
\mathrm{T}_{\mathrm{n}}=\frac{\partial \Phi}{\partial \delta_{\mathrm{n}}}=\frac{\sigma(\lambda)}{\lambda} \frac{\delta_{\mathrm{n}}}{\delta_{\mathrm{n}}^{\mathrm{c}}}, \quad \mathrm{T}_{\mathrm{t}}=\frac{\partial \Phi}{\partial \delta_{\mathrm{t}}}=\frac{\sigma(\lambda)}{\lambda} \frac{\delta_{\mathrm{n}}^{\mathrm{c}}}{\delta_{\mathrm{t}}^{\mathrm{c}}} \frac{\delta_{\mathrm{t}}}{\delta_{\mathrm{t}}^{\mathrm{c}}}
$$

The traction law under a pure normal separation $\left(\delta_{t}=0\right)$ is $T_{n}=\sigma(\lambda)$ where $\lambda=\delta_{n} / \delta_{n}^{c}$; while under a pure tangential displacement $\left(\delta_{n}=0\right), T_{t}=\left(\delta_{n}^{c} / \delta_{t}^{c}\right) \sigma(\lambda)$ where $\lambda=\delta_{t} / \delta_{t}^{c}$. The peak normal traction under pure normal separation is $\hat{\sigma}$ and the peak shear traction is $\left(\delta_{\mathfrak{n}}^{\mathfrak{c}} / \delta_{\mathfrak{t}}^{c}\right) \hat{\sigma}$ in a pure tangential displacement. The work of separation per unit area of interface $\Gamma_{0}$ is given by (2.9) with $\lambda=1$. For the relation $\sigma(\lambda)$ specified in Fig. 1 


$$
\Gamma_{0}=\frac{1}{2} \hat{\sigma} \delta_{\mathrm{n}}^{\mathrm{c}}\left[1-\lambda_{1}+\lambda_{2}\right]
$$

It has been found in [1] that the resistance curve behaviour is rather insensitive to variations of the shape of $\sigma(\lambda)$, for given values of $\hat{\sigma}$ and $\Gamma_{0}$.

A modification of this traction-separation law to incorporate an effect of plastic straining on the failure mechanism is introduced here in a manner analogous to that employed in [3]. With this modification the peak stress $\hat{\sigma}$ in the traction-separation relation of Fig. 1 is gradually reduced when the effective plastic strain $\epsilon_{\mathrm{e}}^{\mathrm{P}}$ along the crack path has exceeded a critical value $\epsilon_{\mathrm{c}}$

$$
\hat{\sigma}= \begin{cases}\hat{\sigma}_{0} & , \text { for } \epsilon_{\mathrm{e}}^{\mathrm{P}} \leq \epsilon_{\mathrm{c}} \\ \hat{\sigma}_{0}-\Delta \hat{\sigma}\left(\epsilon_{\mathrm{e}}^{\mathrm{P}}-\epsilon_{\mathrm{c}}\right) / \Delta \epsilon & , \text { for } \epsilon_{\mathrm{c}}<\epsilon_{\mathrm{e}}^{\mathrm{P}}<\epsilon_{\mathrm{c}}+\Delta \epsilon \\ \hat{\sigma}_{0}-\Delta \hat{\sigma}, & , \text { for } \epsilon_{\mathrm{e}}^{\mathrm{P}} \geq \epsilon_{\mathrm{c}}+\Delta \epsilon\end{cases}
$$

Thus, with (2.12) the cohesive zone model accounts for a reduction of the material strength, which could result from plastic strain controlled nucleation of voids at the interface or from accelerated void growth near the crack tip.

By using (2.4) a reference stress intensity is defined as

$$
K_{0}=\left[\frac{1-v^{2}}{E}+\frac{1-v_{s}^{2}}{E_{s}}\right]^{-1 / 2}\left(\frac{2 \Gamma_{0}}{1-\beta^{2}}\right)^{1 / 2}
$$

Thus, $\mathrm{K}_{0}$ represents the value of $|\mathrm{K}|$ needed to advance the interface crack in the absence of any plasticity. A reference length $R_{0}$, which scales with the size of the plastic zone (when $|K| \simeq K_{0}$ ), is defined as

$$
R_{0}=\frac{1}{3 \pi}\left(\frac{K_{0}}{\sigma_{Y}}\right)^{2}=\frac{2}{3 \pi}\left[\frac{1-v^{2}}{E}+\frac{1-v_{s}^{2}}{E_{s}}\right]^{-1} \frac{\Gamma_{0}}{\left(1-\beta^{2}\right) \sigma_{Y}^{2}}
$$

Since $R_{0}$ also scales with the fracture process zone length, it is natural to define another measure of mixity, $\psi_{0}$, based on the reference length $\mathrm{R}_{0}$ rather than $\mathrm{L}$ in (2.5). By (2.7), $\psi$ and $\psi_{0}$ are related by

$$
\psi_{0}=\psi+\epsilon \ln \left(\mathrm{R}_{0} / \mathrm{L}\right)
$$

In the numerical analyses finite strains are accounted for, using a convected coordinate, Lagrangian formulation of the field equations, in which $\mathrm{g}_{\mathrm{ij}}$ and $\mathrm{G}_{\mathrm{ij}}$ are metric tensors in the reference configuration and the current configuration, respectively, with determinants $g$ and $G$, and $\eta_{i j}=1 / 2\left(G_{i j}-g_{i j}\right)$ is the Lagrangian strain tensor. The contravariant components $\tau^{\mathrm{ij}}$ of the Kirchhoff stress tensor on the current base vectors are related to the components of the Cauchy stress tensor $\sigma^{\mathrm{ij}}$ by $\tau^{\mathrm{ij}}=\sqrt{\mathrm{G} / \mathrm{g}} \mathrm{\sigma}^{\mathrm{ij}}$. Then, in the finite-strain generalization of $\mathrm{J}_{2}$-flow theory discussed by Hutchinson [16], an incremental stress-strain relationship is obtained of the form $\dot{i}^{\mathrm{ij}}=\mathrm{L}^{\mathrm{ijk} \mathrm{l}} \dot{\eta}_{\mathrm{kl}}$. The value of the tangent modulus at a given stress level is determined from the uniaxial true stress-logarithmic strain curve, which is taken to be specified by the power law

$$
\epsilon= \begin{cases}\sigma / \mathrm{E} & , \text { for } \sigma \leq \sigma_{\mathrm{Y}} \\ \left(\sigma_{\mathrm{Y}} / \mathrm{E}\right)\left(\sigma / \sigma_{\mathrm{Y}}\right)^{1 / \mathrm{N}} & , \text { for } \quad \sigma \geq \sigma_{\mathrm{Y}}\end{cases}
$$

Here, $\sigma_{\mathrm{Y}}$ is the initial yield stress, $\mathrm{E}$ is Young's modulus, and $\mathrm{N}$ is the strain hardening exponent.

The computations here are carried out for the case of a rigid lower half-space $\left(E_{s} \rightarrow \infty\right)$, so that only the upper half-space needs to be analysed numerically. With the plastic strain dependent cohesive zone model considered here there is an interest in more crack growth, relative to $R_{0}$, than that considered in [12], and thus a relatively long uniform mesh region is needed in front of the initial crack tip. The mesh used is illustrated in Fig. 2, with outer radius $A_{0}$ and with the element size $\Delta_{0}$ in the uniform mesh region. The results to be shown 


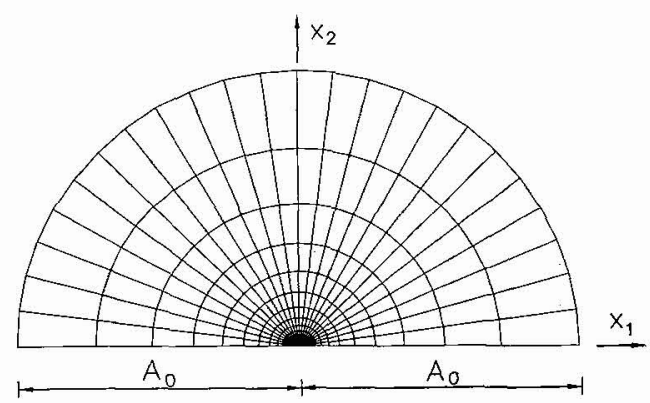

(a)

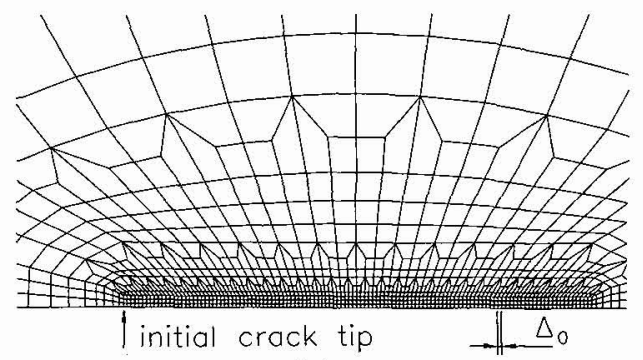

(b)

Fig. 2. Finite element mesh used to analyse interface crack growth. (a) Region analysed numerically. (b) Refined mesh along the crack line.

here are obtained for $A_{0}=8000 \Delta_{0}$, with the value of $\Delta_{0} / R_{0}$ ranging from 0.116 to 0.103 . Apart from the different mesh, the analyses are carried out as described in [1,12]. Thus, for a chosen value of $\psi$ the amplitude $|\mathrm{K}|$ of the displacements on the outer edge are increased incrementally, and a special Rayleigh-Ritz finite element method is employed to control nodal displacements within the fracture process zone (see also [15]).

The elastic-plastic material above the interface (for $x^{2}>0$ ) is taken to be characterized by the values $\sigma_{\mathrm{Y}} / \mathrm{E}=0.003, v=1 / 3$ and $\mathrm{N}=0.1$. Then, with the rigid lower half-space the values of the parameters defined by (2.3) and (2.2) are $\beta=-1 / 4$ and $\epsilon=0.0813$. Thus, with a non-zero value of $\epsilon$ the expression (2.10) does give a shift between $\psi$ and $\psi_{0}$.

For the cohesive zone model $(2.8)-(2.12)$ the parameter values used in the computations are $\delta_{\mathfrak{t}}^{c} / \delta_{\mathfrak{n}}^{c}=1, \lambda_{1}=0.15, \lambda_{2}=0.5$ and $\delta_{n}^{c}=0.05 \Delta_{0}$. Furthermore, in (2.12) various values of the critical strain $\epsilon_{\mathrm{c}}$ are considered, with $\Delta \epsilon=0.05$ and $\left(\hat{\sigma}_{0}-\Delta \hat{\sigma}\right) / \sigma_{\mathrm{Y}}=1.0$. The value of the effective plastic strain $\epsilon_{\mathrm{c}}^{\mathrm{P}}$ in (2.12) is calculated as the average over the quadrilateral element adjacent to the point considered in the debonding region.

\section{RESULTS}

The analyses of interface crack growth in [12] focussed on values of $\hat{\sigma}_{0} / \sigma_{\mathrm{Y}}$ ranging from 1.4 to 3.0 . For $\hat{\sigma}_{0} / \sigma_{Y}=1.4$ the steady-state interface toughness $|K|_{s s}$ was found identical to $K_{0}$ in a range of $\psi_{0}$ values between about $-20^{\circ}$ and $20^{\circ}$, so that here plastic yielding does not contribute to the fracture toughness. For $\hat{\sigma}_{0} / \sigma_{\mathrm{Y}}=2.0$ the value of $\mid \mathrm{K}_{s s} / \mathrm{K}_{0}$ corresponding to $\psi_{0}=0^{\circ}$ is only slightly above unity, while for $\hat{\sigma}_{0} / \sigma_{Y}=3.0$ this value of $\mid \mathrm{KI}_{\mathrm{ss}} / \mathrm{K}_{0}$ is about 2.1. For all values of $\hat{\sigma}_{0} / \sigma_{\mathrm{Y}}$ it was found that the predicted 


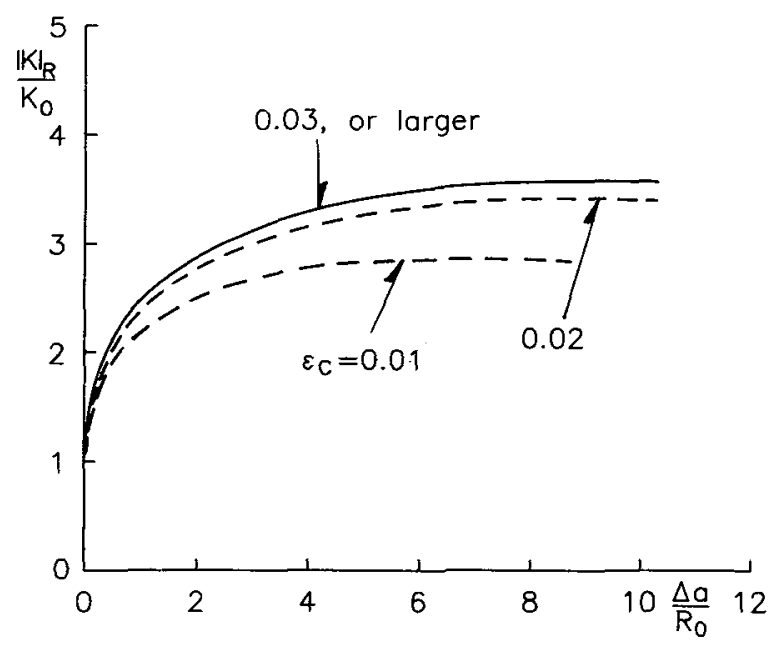

Fig. 3. Interface crack growth resistance curves for $\hat{\sigma}_{0} / \sigma_{Y}=3.4$ and $\psi_{0}=-1.88^{n}$.

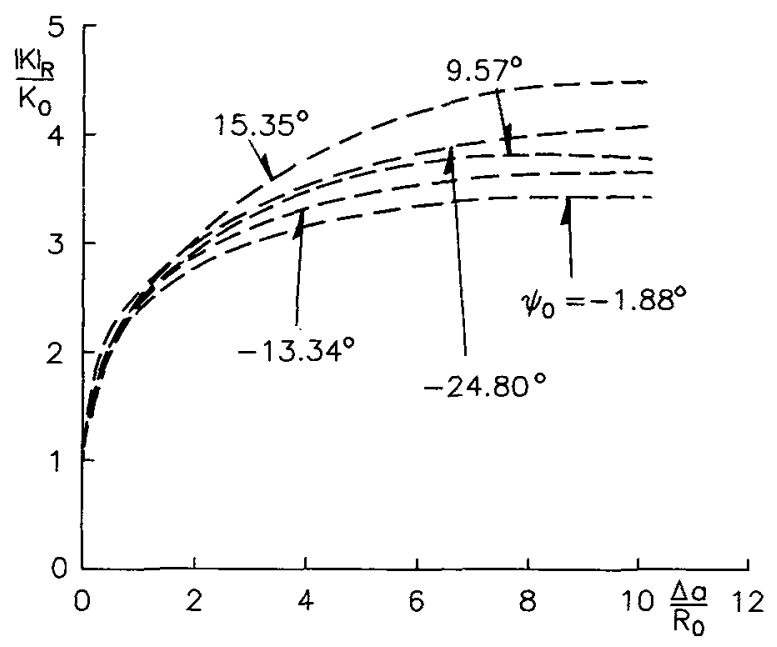

Fig. 4. Interface crack growth resistance curves for $\hat{\sigma}_{0} / \sigma_{Y}=3.4$ and $\epsilon_{\mathrm{c}}=0.02$.

fracture toughness increases significantly with increasing proportion of mode 2 to mode 1 , i.e. for increasing values of $\left|\psi_{0}\right|$. This trend had also been found experimentally $[8,9,10,11]$.

Here, similar results are computed, taking into account the modification (2.12) of the traction-separation law to incorporate an effect of plastic straining on the failure mechanism. Fig. 3 shows resistance curves computed for $\hat{\sigma}_{0} / \sigma_{Y}=3.4$, with $\psi_{0}=-1.88^{\circ}$. The solid curve is predicted using the traction-separation law also used in [12], with no effect of plastic straining, and this resistance curve is indistinguishable from that obtained by using (2.12), with $\epsilon_{\mathrm{c}}=0.03$. The dashed curves, predicted for $\epsilon_{\mathrm{c}}=0.02$ and $\epsilon_{\mathrm{c}}=0.01$, show that the effect of plasticity on interface failure tends to reduce the fracture toughness, analogous to the results found in $[3,4]$ for the case of mode 1 crack growth in a homogeneous material.

In Fig. 4 resistance curves for different values of the mode mixity parameter $\psi_{0}$ are compared, corresponding to fixed values of the material parameters in the traction-separation law, $\hat{\sigma}_{0} / \sigma_{\mathrm{Y}}=3.4$ and $\epsilon_{\mathrm{c}}=0.02$, etc. Thus, the curve for $\psi_{0}=-1.88^{\circ}$ in Fig. 4 is identical to that for $\epsilon_{\mathrm{c}}=0.02$ in Fig. 3. It is seen in Fig. 4 that lowest fracture toughness predicted is found for $\psi_{0}=-1.88^{\circ}$, while for smaller or larger 


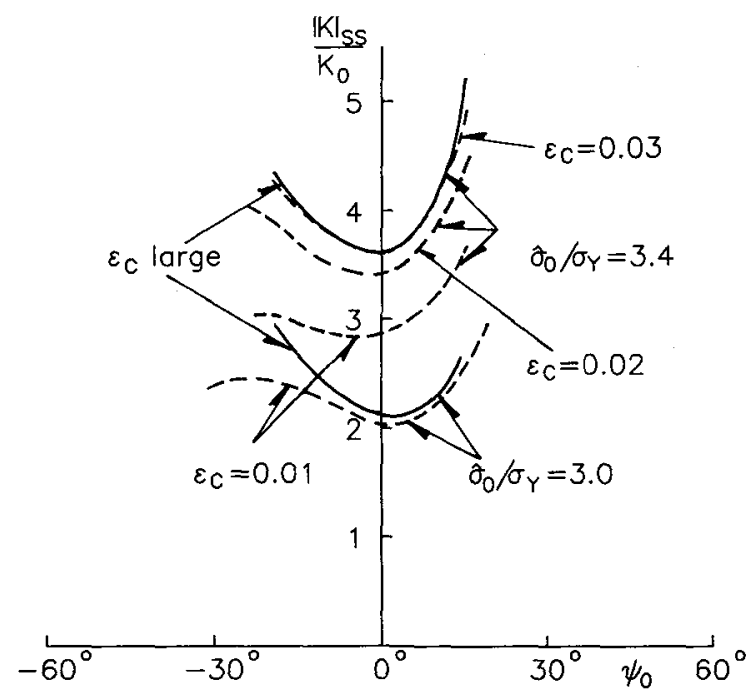

Fig. 5. Steady-state interface toughness as a function of the local mixity measure $\psi_{0}$.

values of $\psi_{0}$, i.e. an increasing proportion of mode 2 to mode 1 , the resistance curves show higher toughness.

Results of many resistance curve analyses, including those illustrated in Figs. 3 and 4, are shown in Fig. 5. Here, the steady-state toughnesses $\mid \mathrm{KI}_{\mathrm{ss}} / \mathrm{K}_{0}$ are plotted as a function of $\psi_{0}$, with each curve representing a particular set of parameters in the modified traction-separation relation (2.12). The solid curve for $\hat{\sigma}_{0} / \sigma_{Y}=3.0$ and $\epsilon_{\mathrm{c}}$ large was also shown in [12], but has been recomputed here, using the mesh in Fig. 2. For comparison with this curve a set of computations have been carried out with a rather low value, $\epsilon_{\mathfrak{c}}=0.01$, of the critical strain for failure. The comparison shows that for all values of $\psi_{0}$ considered the fracture toughness is reduced by the effect of plastic straining in (2.12), and the trend found in [12] is maintained that the lowest fracture toughness occurs at a value of $\psi_{0}$ near $0^{\circ}$, while mixed mode conditions near the crack tip gives higher fracture toughness. This picture starts to break down at $\psi_{0} \simeq-25^{\circ}$, where the curve shows a maximum, so that the toughness starts to decay for larger negative values of $\psi_{0}$. As was also emphasized in [12], curves as those shown in Fig. 5 are not symmetric, but it should be mentioned that the dashed curve for $\hat{\sigma}_{0} / \sigma_{Y}=3.0$ has also a maximum for positive values of $\psi_{0}$, in the range not shown in the figure (around $40^{\circ}$ ). These maxima indicate that plastic yielding is getting dominated by shear along the interface, so that the modification (2.12) of the traction-separation relation is less realistic, as will be further discussed below.

The four curves for $\hat{\sigma}_{0} / \sigma_{\mathrm{Y}}=3.4 \mathrm{in}$ Fig. 5 are those also illustrated by the resistance curves in Figs. 3 and 4. The dashed curve for $\epsilon_{\mathfrak{c}}=0.03$ is not distinguishable from the solid curve in Fig. 3, but it seen in Fig. 5 that this curve drops slightly below the corresponding solid curve as the value of $\psi_{0}$ differs increasingly from $0^{\circ}$. The curves for $\epsilon_{\mathrm{c}}=0.02$ or $\epsilon_{\mathrm{c}}=0.01$ illustrate that an effect of plastic straining on the failure mechanism can give a significant reduction of the fracture toughness. If similar computations were carried out for much higher values of $\hat{\sigma}_{0} / \sigma_{Y}$ than 3.4 , a steady-state fracture toughness for large $\epsilon_{\mathrm{c}}$, as that illustrated by the solid curves in Fig. 5, would not be available for comparison; but based on the mode 1 results obtained in [3] it is expected that a steady state fracture toughness would be predicted when the plastic strain dependent failure mechanism is accounted for. It is noted that crack growth predictions based on the modified traction-separation relation (2.12) will display some mesh dependence, since the plastic strain near the tip is sensitive to the size of the finite elements along the line of the crack.

The predicted maxima on the two curves for $\epsilon_{\mathrm{c}}=0.01$ in Fig. 5, at values of $\psi_{0}$ around $-20^{\circ}$ to $-25^{\circ}$, may be better understood by looking at crack growth predictions, which are directly based on models 
for the nucleation and growth of voids (e.g. Needleman and Tvergaard [17,18], Rousselier [19], and Xia et al. [20]). Clearly, voids grow with plastic straining, but the rate of growth is strongly amplified by increasing levels of the mean stress $\sigma_{m}$, which is not accounted for in (2.12). To incorporate some of this effect in the cohesive zone model $\epsilon_{\mathrm{e}}^{\mathrm{P}}$ in (2.12) could be replaced by $\mathrm{F}$, where $\dot{\mathrm{F}}=\dot{\epsilon}_{\mathrm{e}}^{\mathrm{P}} \mathrm{C} \sigma_{\mathrm{m}} / \sigma_{\mathrm{Y}}$ with a material parameter $C$, and $F=\int \dot{F} d t$. In the present paper, where further modifications of (2.12) along these lines have not been investigated, the model predictions are expected to be reasonable as long as the proportion of mode 2 to mode 1 is moderate.

\section{References:}

[1] Tvergaard V., Hutchinson J.W., J. Mech. Phys. Solids 40 (1992) 1377-1397.

[2] Tvergaard V., Hutchinson J.W., Int. J. Solids Structures 31 (1994) 823-833.

[3] Tvergaard V., Hutchinson J.W., Int. J. Solids Structures 33 (1996) 3297-3308.

[4] Tvergaard V., "Relations between crack growth resistance and fracture process parameters under large scale yielding”, Proc. IUTAM Symposium on Nonlinear Analysis of Fracture, Cambridge University (to appear).

[5] Tvergaard V., Int. J. Mech. Sci. 34 (1992) 635-649.

[6] Tvergaard V., Int. J. Fracture 72 (1995) 277-292.

[7] Paris P.C., Tada H., Zahoor A., Ernst H., The theory of instability of the tearing mode of elastic-plastic crack growth (Elastic-Plastic Fracture, ASTM STP 668, American Society of Testing Materials, 1979) pp. 5-36.

[8] Cao H.C., Evans A.G., Mech. Mater. 7 (1989) 295.

[9] Liechti K.M., Chai, Y.S., J. Appl. Mech. 59 (1992) 295.

[10] Thouless M.D., Acta Met. Mater. 38 (1990) 1135.

[11] O'Dowd N.P., Stout M.G., Shih C.F., Phil. Mag. A66 (1992) 1037.

[12] Tvergaard V., Hutchinson J.W., J. Mech. Phys. Solids 41 (1993) 1119-1135.

[13] Rice J.R., J. Appl. Mech. 55 (1988) 98.

[14] Needleman A., J. Appl. Mech. 54 (1987) 525-531.

[15] Tvergaard V., Materials Science and Engineering A125 (1990) 203-213.

[16] Hutchinson J.W., Finite strain analysis of elastic-plastic solids and structures (Numerical Solution of Nonlinear Structural Problems, American Society for Mechanical Engineers, New York, 1973) p. 17.

[17] Needleman A., Tvergaard V., J. Mech. Phys. Solids 35 (1987) 151-183.

[18] Needleman A., Tvergaard V., Int. J. Fracture 49 (1991) 41-67.

[19] Rousselier G., Nuclear Engng. and Design 105 (1987) 97-111.

[20] Xia L., Shih C.F., Hutchinson J.W., J. Mech. Phys. Solids 43 (1995) 389-413. 